\title{
A new case of Wolbachia dependence in the genus Asobara: evidence for parthenogenesis induction in Asobara japonica
}

\author{
N Kremer ${ }^{1}$, D Charif ${ }^{1}, \mathrm{H}$ Henri $^{1}$, M Bataille ${ }^{1}$, G Prévost ${ }^{2}$, K Kraaijeveld ${ }^{3}$ and F Vavre ${ }^{1}$ \\ ${ }^{1}$ Université de Lyon, F-69000, Lyon; Université Lyon 1; CNRS, UMR5558, Laboratoire de Biométrie et Biologie Evolutive, F-69622, \\ Villeurbanne, France; ${ }^{2}$ Laboratoire de Biologie des Entomophages (EA 3900), Université de Picardie Jules Verne, F-80039, Amiens, France \\ and ${ }^{3}$ Department of Animal Ecology, Institute of Biology Leiden, Leiden University, Leiden, The Netherlands
}

\begin{abstract}
Wolbachia is a maternally inherited bacterium that is widely distributed among arthropods, in which it manipulates the reproduction of its hosts. Although generally facultative for its hosts, Wolbachia has recently become obligatory in Asobara tabida (Hymenoptera: Braconidae) in which it is required for the completion of oogenesis. Here, we describe a new Wolbachia strain (wAjap) that is associated with the genus Asobara and infects Asobara japonica. wAjap was detected in all female-biased populations of $A$. japonica found in the main islands of Japan, but not in the arrhenotokous populations from the southern islands. Using phylogenetic analyses based on multi-locus sequence typing (MLST), we show that this strain is closely related to wAtab3 (the strain required for oogenesis in $A$. tabida), even though they differ on Wolbachia surface protein (WSP) and WO phage sequences. Using antibiotic treatments, we show that cured thelytokous females are not dependent on Wolbachia for
\end{abstract}

oogenesis. However, they produced only sons, showing that wAjap induces thelytokous parthenogenesis. Analyses of mating behavior and offspring production of individuals from Wolbachia-infected populations showed that while males were still sexually functional, females no longer attract males, making Wolbachia an obligate partner for daughter production in thelytokous populations. The fact that Wolbachia has become independently obligatory in two species of the same genus tends to show that dependence evolution can be common and swift, although no clear benefit for the parasitoid can be attributed to this dependence. Although dependence should lead to co-divergence between Wolbachia and its hosts, the very few cases of co-speciation observed in host-Wolbachia associations question the stability of these obligatory associations.

Heredity (2009) 103, 248-256; doi:10.1038/hdy.2009.63; published online 10 June 2009

Keywords: Wolbachia; Asobara; thelytokous parthenogenesis; dependence; symbiosis

\section{Introduction}

Wolbachia (Anaplasmataceae) is a cytoplasmically inherited bacterium that infects a wide variety of hosts ranging from arthropods (Werren, 1997) to nematodes (Bandi et al., 2001). The invasion of this bacterium into arthropod populations is because of its ability to manipulate host reproduction. Four major types of host manipulation by Wolbachia are known: cytoplasmic incompatibility (CI), feminization, male killing and parthenogenesis induction (PI). These effects either decrease the offspring production of uninfected females or bias the sex ratio of infected females toward females. Both result in the enhanced transmission of Wolbachia, as Wolbachia is maternally transmitted through its host oocytes. The more widespread effect is CI, which is found in all major orders of insects, and also in mites and

Correspondence: N Kremer, Université de Lyon, F-69000, Lyon; Université Lyon 1, CNRS, UMR5558, Laboratoire de Biométrie et Biologie Evolutive, F-69622, Villeurbanne, France.

E-mail: natacha.kremer@normalesup.org

Received 14 November 2008; revised 16 March 2009; accepted 3 May 2009; published online 10 June 2009 isopods (Werren, 1997). Feminization of genetic males into functional neo-females is mainly observed in isopod crustaceans (Bouchon et al., 1998), but also occurs in Lepidoptera and Hemiptera (Kageyama et al., 2002; Negri et al., 2006). Male killing independently appeared in phylogenetically unrelated insect groups, such as Lepidoptera and Coleoptera, in which high levels of competition between siblings occurs (Hurst and Jiggins, 2000). PI is often associated with Wolbachia infection in haplodiploid species, especially mites and Hymenoptera (Huigens and Stouthamer, 2003). In uninfected haplodiploids, males develop from unfertilized haploid eggs, whereas females develop from fertilized diploid eggs (arrhenotoky). In contrast, only diploid females are produced in thelytokous reproduction. When thelytoky is Wolbachia-induced, it usually results from the diploidization of non-fertilized eggs during the first mitotic division (Stouthamer et al., 1990; Stouthamer and Kazmer, 1994, but see Weeks and Breeuwer, 2001).

All these reproductive manipulations are generally facultative to the host. However, in the case of Wolbachiainduced thelytoky, any genes involved in sexual reproduction can accumulate mutations without being selected against. Thus, the sexual functionality of males 
and females from thelytokous populations is expected to deteriorate over time, culminating in Wolbachia dependence for daughter production, which has been reported in various species (Zchori-fein et al., 1992, 1995; Pijls et al., 1996; Arakaki et al., 2000; Pannebakker et al., 2005).

Another case of Wolbachia dependence occurs in the wasp Asobara tabida (Hymenoptera, Braconidae) in which Wolbachia is necessary for host oogenesis, rendering aposymbiotic females sterile (Dedeine et al., 2001). This obligatory dependence is believed to be because of a loss of host control over oogenesis (Pannebakker et al., 2007). Alternatively, it may be because of an antidote/poison mechanism imposing the 'sterilization of aposymbiotic sisters' (Charlat and Mercot, 2001), similar to the modification/rescue model of CI (Breeuwer and Werren, 1993). In this context, it is interesting to examine the different species of the genus Asobara to (i) characterize the potential infections by Wolbachia and their effect on host reproduction and (ii) determine whether obligatory dependence to Wolbachia occurs more widely in this genus.

In the genus Asobara, A. citri and A. persimilis are uninfected (Dedeine et al., 2005). A. tabida is infected by two CI-inducing bacterial strains (wAtab1 and wAtab2) and a third one (wAtab3) that is essential for oogenesis completion (Dedeine et al., 2001, 2004). A. rufescens is partially infected by a strain closely related to the CI-inducing strain wAtab1 (Dedeine et al., 2005). In a recent survey of larval parasitoids of frugivorous Drosophilidae in Japan, Mitsui et al. (2007) established the geographical distribution of A. japonica. They showed that field sex ratios are typical of arrhenotokous reproduction in three populations on subtropical islands, whereas those of all five populations sampled on the main (northern) islands are highly female biased. This suggests that the latter could be infected by a reproductive manipulator.

Here, we show that thelytokous populations of A. japonica are infected by Wolbachia. We characterize this Wolbachia strain, called wAjap, using phylogenetic analyses based on multi-locus sequence typing (MLST), Wolbachia surface protein (WSP) and WO phage and determine the phenotype it induces on its host. We show that parthenogenesis in northern populations of A. japonica is caused by Wolbachia. We then examine the extent to which sexual function has decayed as a result of prolonged parthenogenesis. Finally, we show that A. japonica is dependent on Wolbachia for female offspring production, but in contrast to A. tabida it can complete oogenesis in the absence of the symbiont.

\section{Materials and Methods}

\section{Biological system}

Asobara japonica (Hymenoptera:Braconidae:Alysiinae) is a solitary endoparasitoid laying its eggs into the first or second instar larvae of Diptera and especially Drosophila species (Mitsui et al., 2007; Ideo et al., 2008). Wasps were trapped in the field using banana and natural fruits found in some localities (see Mitsui et al. 2007). Wasps from mass-reared lines were kindly provided by MT Kimura. A thelytokous population from Sapporo (Japan) and an arrhenotokous population from Amami-Oshima
(Japan) were maintained at $20^{\circ} \mathrm{C}$, under $70 \%$ relative humidity and 12/12 light/dark (LD) cycle on Wolbachiafree $D$. melanogaster reared on axenic nutritive medium (David, 1962). In the following, $\mathrm{T}(\mathrm{w})$ and $\mathrm{T}(0)$ will refer to infected and uninfected individuals from the thelytokous population, respectively, and A(0) to uninfected individuals from the arrhenotokous population.

\section{Molecular characterization of symbionts and host}

Wasps were individually crushed for $30 \mathrm{~s}(25 \mathrm{~Hz})$ in $150 \mu \mathrm{l}$ of $5 \%$ Chelex solution (Bio-Rad, Hercules, CA, USA) using a TissueLyser (Qiagen, Hilden, Germany). Samples were kept at $56{ }^{\circ} \mathrm{C}$ overnight and then for $15 \mathrm{~min}$ at $95^{\circ} \mathrm{C}$ before centrifugation.

PCRs were carried out in $25 \mu \mathrm{l}$ volumes containing $1 \times$ Taq Buffer, $200 \mu \mathrm{M}$ dNTP, $1.5 \mathrm{mM} \mathrm{MgCl}_{2}, 200 \mathrm{nM}$ primers, 0.5 IU Taq DNA polymerase (Eurobio, Les Ulis, France) and $2 \mu \mathrm{l}$ of DNA solution. PCR was carried out using the thermocycler PTC-100 (MJ Research Inc., Waltham, MA, USA). Thermal cycling conditions were $1 \mathrm{~min}$ at $95^{\circ} \mathrm{C}$, a total of 35 cycles of $30 \mathrm{~s}$ at $95^{\circ} \mathrm{C}, 1 \mathrm{~min}$ at the specific Tm (melting temperature for each primer set shown in Supplementary file S1), $1 \mathrm{~min} 30 \mathrm{~s}$ at $72^{\circ} \mathrm{C}$, and a final extension step of $10 \mathrm{~min}$ at $72{ }^{\circ} \mathrm{C}$.

The Asobara species were characterized by sequencing the ITS2 gene (Internal Transcribed Spacer 2) and Wolbachia by sequencing a set of genes for MLST (Baldo et al., 2006). In the case of $A$. tabida, which naturally harbors three different Wolbachia strains, only the strain, wAtab3 that has been isolated was characterized (Dedeine et al. 2004). In addition to MLST typing, Wolbachia were characterized by sequencing the WSP gene (Wolbachia Surface Protein). The WO_B phage was characterized by sequencing the ORF7 gene (Gavotte et al., 2004). DNA sequences have been deposited in the EMBL database under accession numbers FM872332FM872352.

We tested for the presence of the other endosymbionts Arsenophonus, Cardinium, Rickettsia, Hamiltonella and Spiroplasma by diagnostic PCR (Supplementary file S1).

\section{Phylogenetic analysis}

Additional MLST and WSP sequences used in this study were derived from the Wolbachia MLST database 2-07-1 (http://pubmlst.org/wolbachia/). MLST and WSP sequences are available for 37 Wolbachia strains in this database, including 35 strains belonging to supergroup A or B (Baldo et al., 2006). Sequences are accessible from the GenBank database under accession numbers DQ842268-DQ842486.

Characteristics of sequences used for phylogenetic analyses are summarized in Supplementary file S2. Sequences were aligned using Muscle software (Edgar, 2004). Nucleotides from the four hyper variable regions (HVRs) (positions: 66-156, 192-288, 342-444, 498-596) were used for WSP reconstruction. With regard to the nucleotide positions used for the other genes, see Baldo et al. (2006) for MLST genes and Gavotte et al. (2004) for ORF7 gene. The PhyML software (Guindon and Gascuel, 2003) was used for tree reconstruction with 500 bootstraps. Phylogenetic trees were built using maximum likelihood (ML) inference, with the appropriate evolution model estimated with MrAIC.pl 1.4.3 (Nylander, 2004). The best likelihood score was evaluated using the 
Akaike Information Criterion. The models selected were GTR + I + G for WSP, and the concatenated MLST gene data set, according to Baldo et al. (2006). With regard to ITS2 and ORF7, the evolution models selected for the reconstruction were GTR $+\mathrm{G}$. All trees were drawn using the Dendroscope software (Huson et al., 2007).

\section{Antibiotic treatments}

Cured $A$. japonica were obtained by antibiotic treatments as in Dedeine et al. (2001). Briefly, $150 \mu \mathrm{l}$ of a $2 \%$ rifampicin (Hoechst) solution were added to $1.5 \mathrm{~g}$ of Drosophila standard diet $\left(2 \mathrm{mg} \mathrm{g}^{-1}\right)$ on which 70 Drosophila eggs were placed. As parasitoid larvae feed on the hemolymph of their Drosophila hosts, the antibiotic was transmitted to the developing wasps, thereby rendering them aposymbiotic. As individuals were exposed to the antibiotic during their larval development, embryos were still infected when their sex was determined. Hence, adult parasitoids emerging after this treatment were uninfected females, that is, $T(0)$ females for thelytokous populations that produced $\mathrm{T}(0)$ males when allowed to reproduce as virgins.

\section{Influence of Wolbachia on parasitoid offspring production and sex ratio}

To investigate whether $A$. japonica is dependent on Wolbachia for oogenesis and the production of daughters in thelytokous populations, we employed the crossgenerational treatment outlined in Figure 1a. In the first generation, $\mathrm{T}(\mathrm{w})$ females were reared with or without antibiotics to obtain $\mathrm{T}(0)$ and $\mathrm{T}(\mathrm{w})$ females, respectively. Both types of females were allowed to oviposit as virgins: on standard diet for $\mathrm{T}(0)$ females to produce $\mathrm{T}(0)$ males,

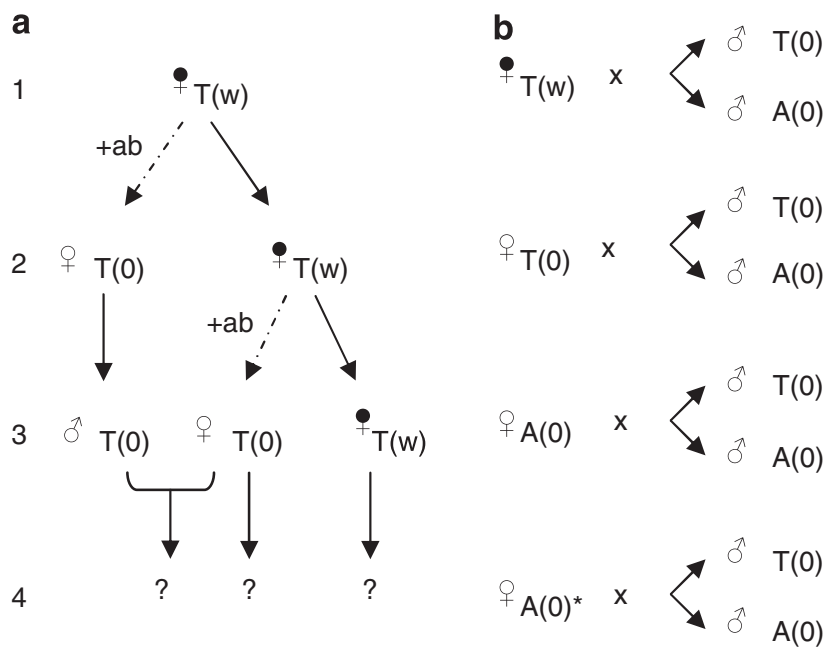

Figure 1 Experimental procedures to determine the phenotypic effect of Wolbachia on A. japonica. (a) Characterization of phenotypic effect of Wolbachia. Offspring production and sex-ratio of each cross were estimated at the fourth generation. (b) Evaluation of sexual degradation. Sexual behavior, latency to courtship, and mating efficiency were estimated for each cross. Full symbols indicate Wolbachia-infected individuals $(\mathrm{w})$. Population of origin is indicated by A (arrhenotokous population from Amami-Oshima) or $\mathrm{T}$ (thelytokous population from Sapporo). Full arrows indicate standard treatment; dotted arrows indicate antibiotic treatment (ab). Star corresponds to an antibiotic treatment carried out on an uninfected female $\mathrm{A}(0)$. on standard diet with or without antibiotics for $\mathrm{T}(\mathrm{w})$ females to produce $\mathrm{T}(0)$ and $\mathrm{T}(\mathrm{w})$ females, respectively. In the third generation, we carried out three different treatments (30 replicates each) with four females allowed to oviposit on 150 Drosophila larvae on standard diet: (i) virgin $\mathrm{T}(\mathrm{w})$ females as a control treatment, (ii) virgin $\mathrm{T}(0)$ females and (iii) $\mathrm{T}(0)$ females with $\mathrm{T}(0)$ males (mating allowed for $48 \mathrm{~h}$ ). For each treatment, the male and female offspring were counted and the sex ratio scored as the percentage of females.

\section{Influence of Wolbachia on oocyte load}

Asobara females are mainly pro-ovogenic and thus emerge with most of their oocytes. The oocyte load of thelytokous and arrhenotokous females was estimated in standard conditions or after antibiotic treatment (that is, $\mathrm{T}(\mathrm{w}), \mathrm{A}(0)$, $\mathrm{T}(0)$ and $\mathrm{A}(0)^{*}$ females, respectively). Newly emerged females were kept for 5 days with water and honey to allow the completion of oocyte maturation. One ovary was dissected in PBS and gently squashed between the slide and the cover glass to disperse its content. Oocytes were then counted under the microscope (AxioCam Imager Z.1, Zeiss, Oberkochen, Germany). Oocyte load was estimated for 20 ovaries for each treatment.

\section{Influence of Wolbachia on host reproduction and sexual behavior}

$\mathrm{T}(0)$ males obtained after antibiotic treatments were placed together with $\mathrm{T}(0)$ females (in mass). Males and females were observed for more than $1 \mathrm{~h}$ during which we scored male courtship (wing vibrations), female receptivity (females stand motionless when males try to copulate) and matings.

In addition, we looked for the presence of spermatozoids in the testes of $\mathrm{T}(0)$ males. Testes were dissected in A-buffer $\left([\mathrm{KCl}]=25 \mathrm{mM},\left[\mathrm{MgCl}_{2}\right]=10 \mathrm{mM}\right.$, [Sucro$\mathrm{se}]=250 \mathrm{mM}$, [Tris] $=35 \mathrm{mM}, \mathrm{pH}=7.5)$, treated with glacial acetic acid $60 \%$ ( $3 \mathrm{~min})$, fixed with Carnoy and mounted in $20 \mu \mathrm{l}$ of Vectashield HardSet containing $1.5 \mu \mathrm{g} \mathrm{ml}^{-1}$ DAPI (4'-6-Diamidino-2-phenylindole, Vector Laboratories, Burlingame, CA, USA). Preparations were observed using a fluorescence microscope (AxioCam Imager Z.1, Zeiss) at the excitation wavelength of $350 \mathrm{~nm}$.

To evaluate the degree of sexual degradation, $\mathrm{T}(\mathrm{w})$, $\mathrm{T}(0)$ and $\mathrm{A}(0)$ females were crossed either with $\mathrm{A}(0)$ or $\mathrm{T}(0)$ males (Figure $1 \mathrm{~b}$ ). To test for possible effects of the antibiotic treatment, we also tested $\mathrm{A}(0)$ females that were treated with antibiotics $\left(\mathrm{A}(0)^{*}\right)$. Ten couples (one male with one female) of each combination were observed for $5 \mathrm{~min}$ to (i) detect possible courtship or mating behaviors and (ii) determine the latency to courtship (if any) evaluated as the time from the start of the exposure to the first wing vibration by the male. Presence of a female in the progeny was checked to determine the sexual functionality of the different crosses.

\section{Results}

Highly female-biased sex ratio is associated with infection by Wolbachia in $A$. japonica populations

Individuals from female-biased populations were all infected by Wolbachia (Sapporo ( $n=6$ females tested), Hirosaki $(n=5)$, Sendai $(n=3)$, Tokyo $(n=5)$ and 
Kagoshima $(n=5))$, whereas individuals from nonbiased populations were never infected (Amami-Oshima $(n=8)$ and Iriomote-Jima $(n=10))$. Sequencing WSP showed infection by a single Wolbachia strain, which we called wAjap. Diagnostic PCR for other classical endosymbionts showed no amplification for Arsenophonus, Cardinium, Rickettsia, Hamiltonella and Spiroplasma.

\section{Phylogenetic position of wAjap}

The phylogenetic relationship between wAjap and other Wolbachia strains was first assessed by using concatenated MLST loci (Figure 2). The Wolbachia strain present in A. japonica (wAjap) clusters with Wolbachia strains infecting other parasitoids of Diptera (A. tabida, Muscidifurax uniraptor and Nasonia vitripennis).

Furthermore, we analyzed Wolbachia strains using the WSP gene (Figure 3). The phylogenetic tree indicates that wAjap was neither directly associated with the Wolbachia strains of $A$. tabida nor with the strains of $M$. uniraptor and $N$. vitripennis (which are grouped together), high- lighting the incongruence between MLST and WSPbased phylogenies, as already shown in Baldo et al. 2005. Analysis of the entire proteic WSP sequence of the four species clustered in MLST allowed detection of mutations at sites under positive selection (Jiggins et al., 2002) and different potential recombination events between hypervariable regions (Supplementary file S3).

In addition, we checked for the presence of WO_B phages and detected two different phages (Supplementary file S4). The Ajap 2 phage was also closely related to the phage Atab_C, known to infect wAtab3, again suggesting that wAjap and wAtab3 might have a recent common ancestor. However, the other phage of wAjap and the two others of wAtab3 are not closely related, suggesting some recombination or phage transfer events such as in WSP.

Finally, the ITS2 gene was sequenced to determine the phylogenetic relationships among the different species of Asobara. The phylogenetic tree reconstructed indicates that $A$. japonica is more related to $A$. persimilis than to the other Asobara species (Figure 4). The topology of this tree

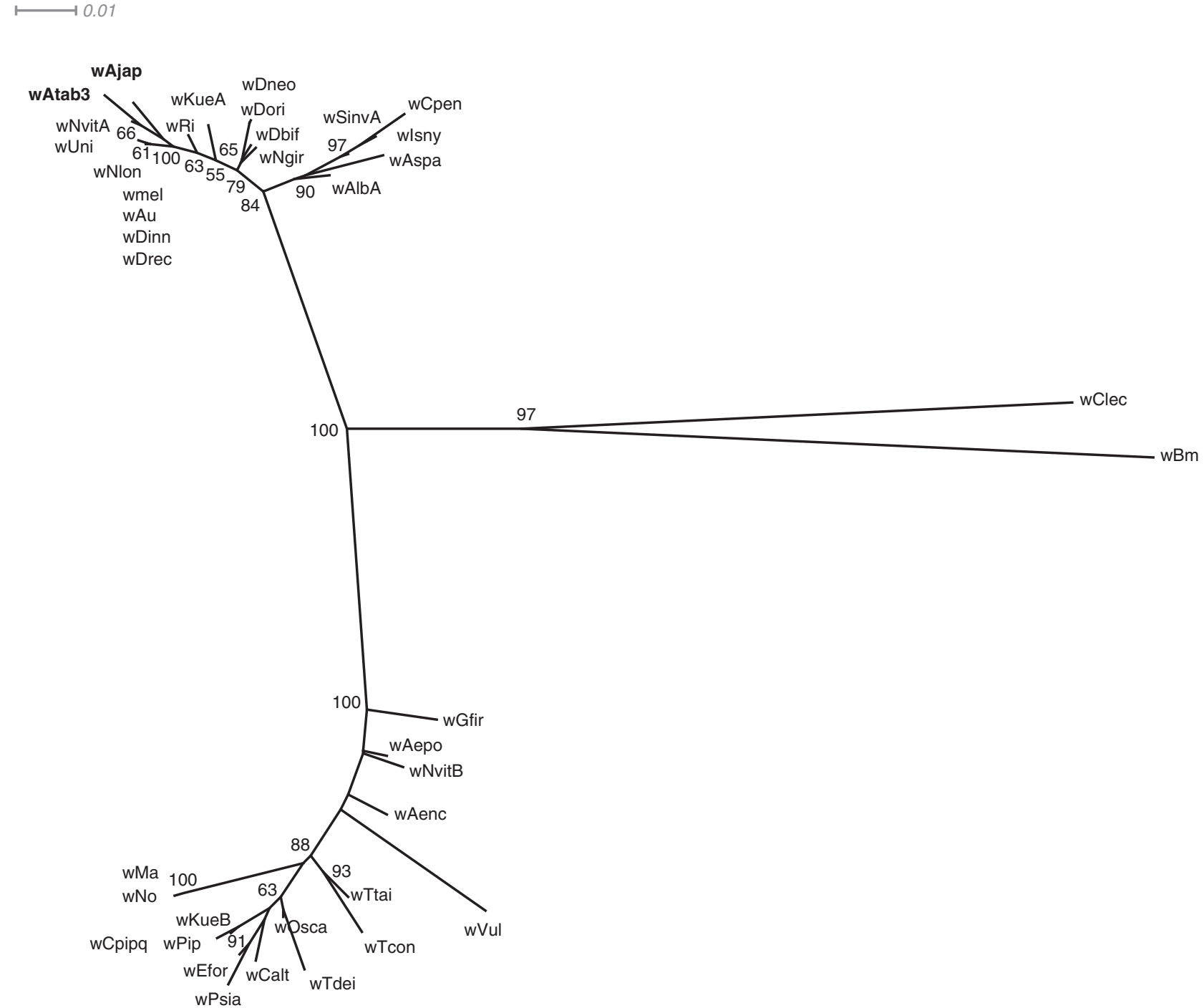

Figure 2 Maximum Likelihood inference phylogeny based on the concatenated sequences of five genes (MLST) of 39 Wolbachia strains. Numbers at tree nodes represent bootstrap support values (500 replicates) higher than 50 . The codes of Wolbachia strains correspond to the name of their host (see Supplementary file S2 for more details). 


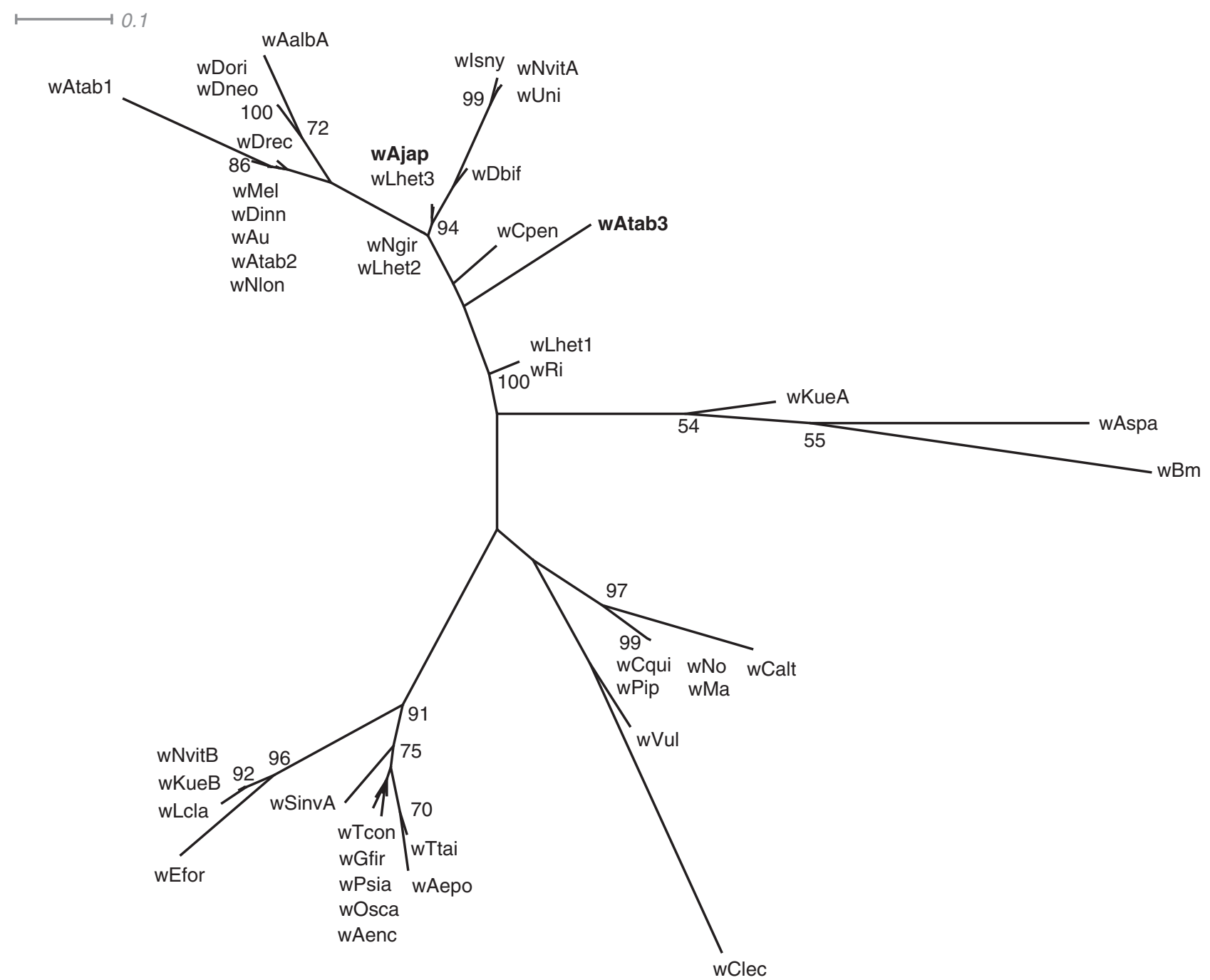

Figure 3 Maximum Likelihood inference phylogeny based on WSP sequences of 44 Wolbachia strains. Numbers at tree nodes represent bootstrap support values (500 replicates) higher than 50. The codes of Wolbachia strains correspond to the name of their host (see Supplementary file S2 for more details).

was congruent with those obtained with $18 S$ gene (data not shown).

\section{Wolbachia induces thelytokous parthenogenesis}

\section{in A. japonica}

To determine the effect of Wolbachia on A. japonica more precisely, we treated females from a female-biased population with antibiotics. As shown in Figure 5, T(0) females produced only males, whereas $T(w)$ females produced mostly females $(S R=0.99 \pm 0.01)$. There was no influence of the infection status on the total offspring production (Kruskal-Wallis rank-sum test; $\chi^{2}=4.31$, d.f. $=2, P$-value $=0.12$ ), suggesting that the variation in sex ratio was not because of preimaginal mortality. Furthermore, quantification of oocyte load showed a slight reduction in $\mathrm{T}(0)$ females $(\mathrm{T}(\mathrm{w})=61.40 \pm 1.14$ vs $\mathrm{T}(0)=54.35 \pm 1.26, t=9.7$, d.f. $=18, P<0.001)$, but this variation was also observed after antibiotic treatment of $\mathrm{A}(0)$ females $\left(\mathrm{A}(0)=63.50 \pm 1.71\right.$ vs $\mathrm{A}(0)^{*}=51.70 \pm 1.75$, $t=11.80$, d.f. $=18, P<0.001)$, suggesting a direct effect of the treatment (ANOVA; treatment, $\mathrm{F}_{1,76}=40.23, P<10^{-7}$; strain, $F_{1,76}=0.03, P=0.85 ; \quad$ Interaction, $F_{1,76}=2.55$, $P=0.11$ ). Consequently, Wolbachia is not necessary for oogenesis in $A$. japonica as opposed to $A$. tabida. All together, these results show that Wolbachia only induces thelytokous parthenogenesis in A. japonica. Finally, $\mathrm{T}(0)$ females produced only sons even when put together with $\mathrm{T}(0)$ males. This can be explained by the complete absence of courtship and mating as observed during the first hour of contact between them. In these populations, Wolbachia is thus obligatory for daughter production.

\section{Females have lost the ability to sexually reproduce,} not males

To estimate the magnitude of sexual degradation in males and females originating from thelytokous populations, we reciprocally crossed arrhenotokous and thelytokous males and females (treated or not with antibiotics) (Figure $1 b, n=10$ for each cross). Females 


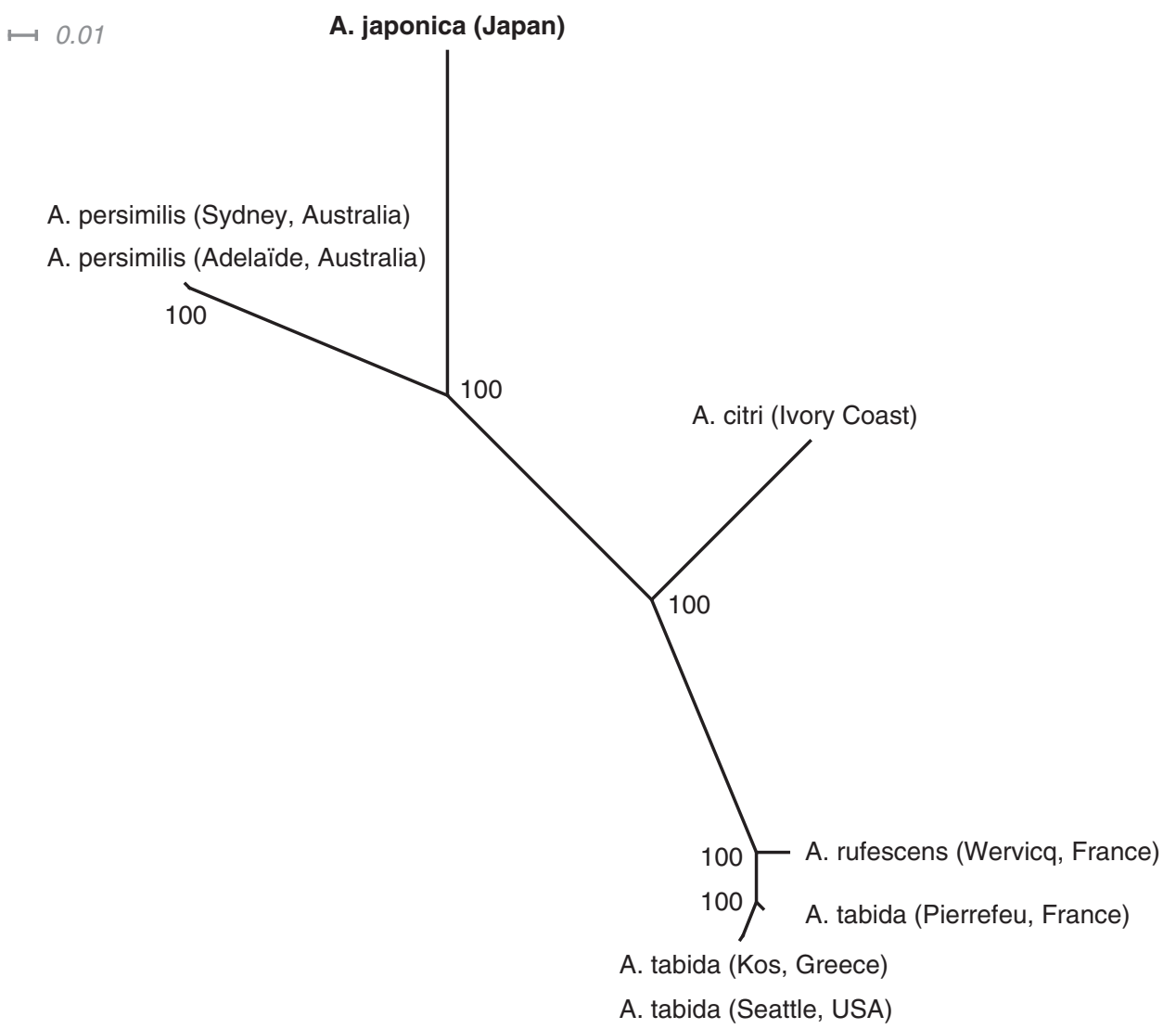

Figure 4 Maximum Likelihood inference phylogeny based on ITS2 sequences of five species of the genus Asobara. Numbers at tree nodes represent bootstrap support values (500 replicates) higher than 50 .
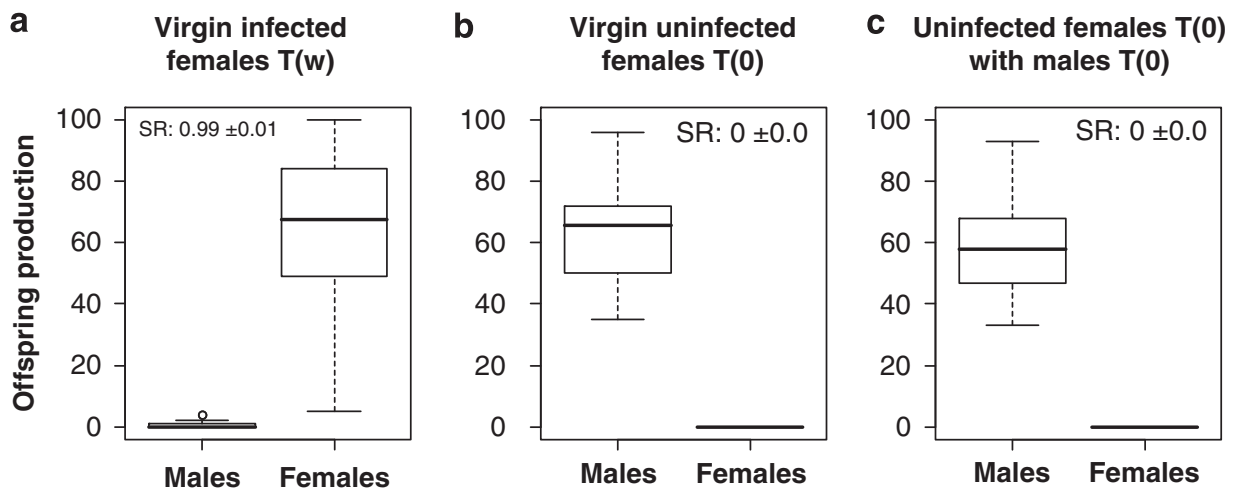

Figure 5 Influence of Wolbachia on female offspring production. Offspring production of (a) infected T(w) females $(n=28)(\mathbf{b})$ virgin uninfected females $\mathrm{T}(0)(n=30)$ (c) uninfected females $\mathrm{T}(0)$ in presence of males $\mathrm{T}(0)(n=30)$. Box-and-whisker plot shows the extreme of the lower whisker, the lower hinge, the median, the upper hinge, and the extreme of the upper whisker for each group.

treated and not treated with antibiotics showed the same pattern regarding courtship behavior (ANOVA, $\mathrm{F}_{1,27}=0.03, P$-value $=0.88$, showing that antibiotic treatment and infection status do not affect the measured traits. These data were then pooled for a graphical representation (Figure 6a). $\mathrm{T}(\mathrm{w})$ or $\mathrm{T}(0)$ females put together with $\mathrm{T}(0)$ and $\mathrm{A}(0)$ males never showed courtship behavior, showing that thelytokous females were not able to reproduce sexually. In contrast to females, $\mathrm{T}(0)$ males courted $\mathrm{A}(0)$ females by showing wing vibration and sometimes complete mating sequence, as efficiently as $\mathrm{A}(0)$ males (Figure $6 \mathrm{a}, \chi_{(2 ; 0.05)}^{2}=3, P=0.22$ ). Latency to courtship was also not significantly different between $\mathrm{A}(0)$ and $\mathrm{T}(0)$ males (ANOVA, $\mathrm{F}_{1,27}=0.22$, $P$-value $=0.64$, Figure $6 \mathrm{~b})$. Furthermore, numerous spermatozoids in differentiation were observed by DAPI staining in the lumen of the testis (Supplementary file S5) in $\mathrm{T}(0)$ males. Finally, $\mathrm{A}(0)$ females crossed with $\mathrm{T}(0)$ males produced daughters (average proportion males $0.27, n=3)$, although relatively fewer than $\mathrm{A}(0)$ males 

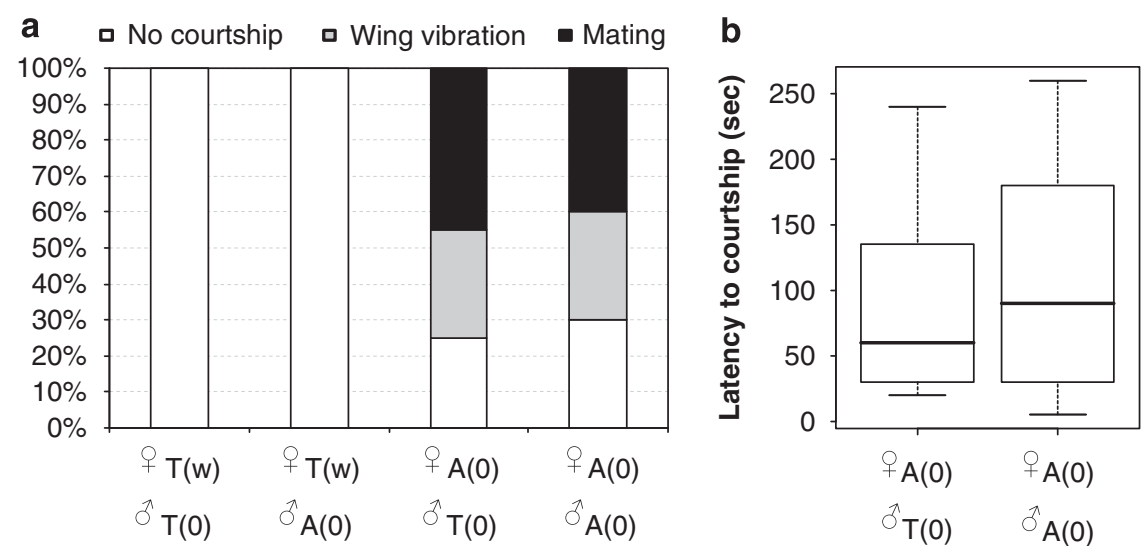

Figure 6 Influence of Wolbachia on sexual behavior degradation. (a) Courtship behavior between arrhenotokous (A) and thelytokous (T) individuals: no courtship behavior (white), wing vibrations (gray), mating (black). $(n=2 \times 10$ for each crossing; antibiotic-treated and untreated females pooled). (b) Latency for thelytokous and arrhenotokous males to court an arrhenotokous female (in seconds). ( $n=6+9$, $8+6$, respectively; antibiotic-treated and untreated females pooled). Population of origin is indicated by A (arrhenotokous population from Amami-Oshima) or T (thelytokous population from Sapporo). Box-and-whisker plot shows the extreme of the lower whisker, the lower hinge, the median, the upper hinge and the extreme of the upper whisker for each group.

(average proportion males $0.17, n=6$, glm with binomial error: deviance $=6.06, P=0.014)$. Hence, males from infected populations are still sexually functional, whereas females are not.

\section{Discussion}

Phylogenetic relationships between Wolbachia strains, host spectrum and induced phenotype

In addition to A. japonica, 66 Hymenoptera species have been reported to be infected by a PI-microorganism. Of these, 46 were infected by Wolbachia (Huigens and Stouthamer, 2003). However, PI-Wolbachia do not form a monophyletic group within the Wolbachia phylogeny (review in Huigens and Stouthamer, 2003), and the same pattern was found using MLST (Baldo et al., 2006).

On the basis of MLST genes, wAjap was phylogenetically close to the Wolbachia strain of A. tabida necessary for oogenesis completion (wAtab3). Similarly, one of the two phages of wAjap was closely related to a phage of wAtab3. These phylogenetic associations might reflect a shared ancestry between A. japonica and A. tabida, although they are not the closest species of the Asobara genus. Alternatively, a recent horizontal transfer event could have occurred as A. japonica and A. tabida are ecologically related and share some of the same Drosophila hosts. Our data do not allow us to differentiate between these hypotheses.

In any case, these closely related strains induce very different effects (PI, oogenesis completion), indicating rapid evolution of an induced phenotype. This result is reinforced by the phylogenetic association between the wAjap and the Wolbachia strains of M. uniraptor (wUni) and N. vitripennis (wNvitA), inducing PI and CI, respectively. Hence, transitions among phenotypes can occur between closely related strains. This could reflect a host effect, as previously described in the Wolbachia strain of Ostrinia scapularis inducing feminization in its natural host, but causing male killing when transferred in Ephestia kuehniella (which is naturally infected by a CI-inducing Wolbachia strain) (Fujii et al., 2001). Similarly, the male-killer, wBol1, was shown to induce CI when infecting male-killing resistant hosts (Hornett et al., 2008).

In contrast to the pattern observed using MLST reconstruction, genetic variations were observed in other parts of the Wolbachia genome (WSP and bacteriophage $\mathrm{WO}$ ), showing that this genome is a mosaic of regions evolving at very different rates. If genes involved in phenotypic effects are rapidly evolving, this could also explain why strains, which are closely related based on MLST, induce different effects.

The close relationship among these four Wolbachia strains is also particularly interesting as all of them are parasitoids of Diptera. It has been suggested that horizontal transfer of Wolbachia is more efficient among closely related species (Bouchon et al., 1998), which could partly explain why these parasitoids are infected with related Wolbachia strains. However, these parasitoids belong to very different families of Hymenoptera and thus other factors, such as sharing of similar hosts, may explain this pattern. This would illustrate how the combination of ecological connections and phylogenetic relatedness of hosts can shape the efficiency of horizontal transmission of Wolbachia.

\section{Induction of parthenogenesis by Wolbachia and its} consequences for sexual reproduction

Infected females produced female offspring without having mated and aposymbiotic females produced only males. Both findings indicate that Wolbachia induces parthenogenesis in A. japonica. Populations of A. japonica in the subtropical islands are sexual, whereas populations in the main Japanese islands are asexual (Mitsui et al., 2007). We have shown that this situation corresponds to the presence of a PI-Wolbachia in the parthenogenetic populations. In addition, reciprocal crosses between individuals from these conspecific allopatric populations allowed us to show that (i) thelytokous males induced by antibiotics from parthenogenetic populations mate with females from sexual populations and produce daughters; (ii) females from parthenogenetic populations are never courted by males. The latter finding suggests a reduction/absence of 
sexual pheromone production in thelytokous females similar to the situation described in Trichogramma cordubensis (Silva and Stouthamer, 1997). Given that females from thelytokous populations from which Wolbachia had been removed still failed to attract matings, the unattractiveness must be because of the changes in the wasp genome, rather than a direct effect of Wolbachia infection. It follows that only thelytokous females are no longer capable of sexual reproduction. Wolbachia is thus obligatory for daughter production in infected populations, therefore rendering the wasp completely dependent on its symbiont.

Wolbachia-induced thelytokous parthenogenesis is generally fixed in natural populations, except in some rare cases in which the effect of Wolbachia is counterbalanced, notably by the presence of other selfish elements (Stouthamer et al., 2001; Huigens and Stouthamer, 2003). So far, it has not been possible to restore sexual reproduction in lines derived from populations in which thelytoky is fixed (review in Pannebakker, 2004). An explanation for this pattern could be that when thelytokous parthenogenesis reaches fixation, genes involved in sexual reproduction are no longer under selection and can accumulate mutations (Carson et al., 1982; Zchori-Fein et al., 1992). However, sexual decay seems stronger in females than in males in A. japonica, as in the three other species in which asexual infected and sexual uninfected populations occur (Apoanagyrus diversicornis, Pijls et al., 1996; Telenomus nawai, Arakaki et al., 2000; Leptopilina clavipes, Pannebakker et al., 2005). This cannot be explained by neutral accumulation of mutations, because these should affect both sexes equally. Additional hypotheses have been proposed. First, some traits involved in female sexual reproduction are likely to be expressed in thelytokous populations. Such traits could be counter-selected if there is a cost to maintain them, like pheromone production (Pijls et al., 1996). Second, fixation of Wolbachia and loss of sexual reproduction could be explained by the 'virginity mutation' hypothesis: in a population partly infected by Wolbachia, mutations allowing females to produce more males (by preventing mating or egg fertilization) will spread rapidly in the population until the mutation and the infection are fixed (Huigens and Stouthamer, 2003; Jeong and Stouthamer, 2005). These mutations can be selected for two reasons. First, infected thelytokous females are still supposed to be able to be fertilized by males and produce females through sexual reproduction in the beginning of the association, as already shown in different Trichogramma species in which both thelytokous and arrhenotokous females coexist (Stouthamer and Kazmer, 1994). Second, infected females produce males when transmission of Wolbachia is not perfect. These two factors allow gene flow between infected and uninfected individuals in the population. In both scenarios, sexual decay in females is selected for, which could lead to a rapid loss of the ability to reproduce sexually, and a swift evolution toward dependence on Wolbachia in these populations.

Independent evolution of Wolbachia dependence in the genus Asobara

In populations of $A$. japonica in which wAjap is fixed, sexual reproduction is no longer possible because of the failure of the thelytokous females to elicit male courtship. In such populations, Wolbachia is necessary for female offspring production. Consequently, $A$. japonica is dependent on Wolbachia in these populations, but not in populations free of infection. The existence within the same species of Wolbachia-free individuals with individuals that are dependent on Wolbachia for reproduction illustrates how rapidly this situation can evolve. In addition, A. japonica is the second species in the genus Asobara showing dependence on Wolbachia, the other being A. tabida, which depends on Wolbachia for oogenesis (Dedeine et al., 2001). It is interesting to note that these two strains are phylogenetically close, but the mechanisms underlying this dependence are completely different and have clearly evolved independently. Again, this illustrates that dependence on Wolbachia can quickly evolve and may occur frequently.

In these two cases, Wolbachia symbiosis is necessary for the host but does not provide a new additional function that would potentially be beneficial to the host, as reproductive functions pre-existed Wolbachia infection. Therefore, the relationship between the wasp and its Wolbachia strain seems to be an obligatory dependence derived from a parasitic association rather than a real mutualistic association. Growing evidence that dependence can evolve rapidly without having a clear mutualistic effect sheds new light on the initial stage of numerous associations between insects and bacteria. Whether mutualism has evolved secondarily to dependence is difficult to show in ancient associations, but it is suggested by these examples in which recent evolution of dependence is not beneficial to the host.

When complete interdependence has evolved, cospeciation between host and symbiont is expected. However, almost all cases of host dependence on Wolbachia are recent at the evolutionary time, and only very few cases of co-speciation occur in host-Wolbachia associations such as in filarial nematodes (Fenn and Blaxter, 2004). This could suggest that although evolution of host dependence can occur frequently and rapidly, these associations are not stable at the evolutionary time scale, either because interdependence is reversible, or because these interactions are more prone to extinction.

\section{Acknowledgements}

We thank $\mathrm{H}$ Mitsui and MT Kimura for supplying different populations of A japonica, $\mathrm{G}$ Gueguen for having confirmed by PCR the absence of other endosymbionts and BA Pannebakker for helpful comments on the paper. This work was supported with funds from UMR CNRS 5558, IFR 41, ANR EndoSymbArt and GDR 2153.

\section{References}

Arakaki N, Noda H, Yamagishi K (2000). Wolbachia-induced parthenogenesis in the egg parasitoid Telenomus nawai. Entomol Exp Appl 96: 177-184.

Baldo L, Lo N, Werren JH (2005). Mosaic nature of the Wolbachia surface protein. J bacteriol 187: 5406-5418.

Baldo L, Dunning Hotopp JC, Jolley KA, Bordenstein SR, Biber SA, Choudhury RR et al. (2006). Multilocus sequence typing system for the endosymbiont Wolbachia pipientis. Appl Environ Microbiol 72: 7098-7110. 
Bandi C, Dunn AM, Hurst DD, Rigaud T (2001). Inherited microorganisms, sex-specific virulence and reproductive parasitism. Trends parasitol 17: 88-94.

Bouchon D, Rigaud T, Juchault P (1998). Evidence for widespread Wolbachia infection in isopod crustaceans: molecular identification and host feminization. Proc Biol Sci 265: 1081-1090.

Breeuwer JAJ, Werren JH (1993). Effect of genotype on cytoplasmic incompatibility between 2 species of Nasonia. Heredity 70: 428-436.

Carson HL, Chang LS, Lyttle TW (1982). Decay of female sexual behavior under parthenogenesis. Science 218: 68-70.

Charlat S, Mercot H (2001). Did Wolbachia cross the border? Trends Ecol Evol 16: 540-541.

David J (1962). A new medium for rearing Drosophila in axenic conditions. Drosophila Info Serv 36: 128.

Dedeine F, Bouletreau M, Vavre F (2005). Wolbachia requirement for oogenesis: occurrence within the genus Asobara (Hymenoptera, Braconidae) and evidence for intraspecific variation in A. tabida. Heredity 95: 394-400.

Dedeine F, Vavre F, Fleury F, Loppin B, Hochberg ME Bouletreau M (2001). Removing symbiotic Wolbachia bacteria specifically inhibits oogenesis in a parasitic wasp. Proc Natl Acad Sci USA 98: 6247-6252.

Dedeine F, Vavre F, Shoemaker DD, Bouletreau M (2004). Intraindividual coexistence of a Wolbachia strain required for host oogenesis with two strains inducing cytoplasmic incompatibility in the wasp Asobara tabida. Evolution Int J Org Evolution 58: $2167-2174$

Edgar RC (2004). MUSCLE: multiple sequence alignment with high accuracy and high throughput. Nucleic Acids Res 32: 1792-1797.

Fenn K, Blaxter M (2004). Are filarial nematode Wolbachia obligate mutualist symbionts? Trends Ecol Evol 19: 163-166.

Fujii Y, Kageyama D, Hoshizaki S, Ishikawa H, Sasaki T (2001). Transfection of Wolbachia in Lepidoptera: the feminizer of the adzuki bean borer Ostrinia scapulalis causes male-killing in the Mediterranean flour moth Ephestia kuehniella. Proc Biol Sci 268: 855-859.

Gavotte L, Vavre F, Henri H, Ravallec M, Stouthamer R, Boulétreau M (2004). Diversity, distribution and specificity of WO phage infection in Wolbachia of four insect species. Insect Mol Biol 13: 147-153.

Guindon S, Gascuel O (2003). A simple, fast, and accurate algorithm to estimate large phylogenies by maximum likelihood. Syst Biol 52: 696-704.

Hornett EA, Duplouy AM, Davies N, Roderick GK, Wedell N, Hurst GD et al. (2008). You can't keep a good parasite down: evolution of a male-killer suppressor uncovers cytoplasmic incompatibility. Evolution 62: 1258-1263.

Huigens ME, Stouthamer R (2003). Parthenogenesis associated with Wolbachia. In: Bourtzis K, Miller TA (eds). Insect Symbiosis, vol. 1. CRC press: Boca Raton, FL, USA. pp 247266.

Hurst GDD, Jiggins FM (2000). Male-killing bacteria in insects: mechanisms, incidence, and implications. Emerg Infect Dis 6: 329-336.

Huson DH, Richter DC, Rausch C, Dezulian T, Franz M, Rupp R (2007). Dendroscope: an interactive viewer for large phylogenetic trees. BMC Bioinformatics 8: 460.

Ideo S, Watada M, Mitsui H, Kimura MT (2008). Host range of Asobara japonica (Hymenoptera: Braconidae), a larval parasitoid of drosophilid flies. Entomol Sci 11: 1-6.
Jeong G, Stouthamer R (2005). Genetics of female functional virginity in the parthenogenesis-Wolbachia infected parasitoid wasp Telenomus nawai (Hymenoptera: Scelionidae). Heredity 94: 402-407.

Jiggins FM, Hurst GD, Yang Z (2002). Host-symbiont conflicts: positive selection on an outer membrane protein of parasitic but not mutualistic Rickettsiaceae. Mol Biol Evol 19: 1341-1349.

Kageyama D, Nishimura G, Hoshizaki S, Ishikawa Y (2002). Feminizing Wolbachia in an insect, Ostrinia furnacalis (Lepidoptera: Crambidae). Heredity 88: 444-449.

Mitsui H, Van Achterberg K, Nordlander G, Kimura MT (2007). Geographical distributions and host associations of larval parasitoids of frugivorous Drosophilidae in Japan. J Nat Hist 41: 1731-1738.

Negri I, Pellecchia M, Mazzoglio PJ, Patetta A, Alma A (2006). Feminizing Wolbachia in Zyginidia pullula (Insecta, Hemiptera), a leafhopper with an $\mathrm{XX} / \mathrm{XO}$ sex-determination system. Proc Biol Sci 273: 2409-2416.

Nylander JAA (2004). MrAIC.pl. Program distributed by the author, Evolutionary Biology Centre, Uppsala University, Sweden.

Pannebakker BA (2004). Evolutionary Consequences of WolbachiaInduced Parthenogenesis in the Parasitoid Leptopilina Clavipes, Leiden University, The Netherlands.

Pannebakker BA, Schidlo NS, Boskamp GJ, Dekker L, van Dooren TJ, Beukeboom LW et al. (2005). Sexual functionality of Leptopilina clavipes (Hymenoptera: Figitidae) after reversing Wolbachia-induced parthenogenesis. J Evol Biol 18: 1019-1028.

Pannebakker BA, Loppin B, Elemans CP, Humblot L, Vavre F (2007). Parasitic inhibition of cell death facilitates symbiosis. Proc Natl Acad Sci USA 104: 213-215.

Pijls JWAM, van Steenbergen HJ, van Alphen JJM (1996). Asexuality cured: the relations and differences between sexual and asexual Apoanagyrus diversicornis. Heredity 76: 506-513.

Silva IMMS, Stouthamer R (1997). To mate or not to mate. Can sex pheromones be used as a taxonomic tool in Trichogramma spp? Proc Exp Appl Entomol NEV Amsterdam 8: 41-46.

Stouthamer R, Kazmer DJ (1994). Cytogenetics of microbeassociated parthenogenesis and its consequences for gene flow in Trichogramma wasps. Heredity 73: 317-327.

Stouthamer R, Luck RF, Hamilton WD (1990). Antibiotics cause parthenogenetic Trichogramma (Hymenoptera/Trichogrammatidae) to revert to sex. Proc Natl Acad Sci USA 87: 2424-2427.

Stouthamer R, van Tilborg M, de Jong JH, Nunney L, Luck RF (2001). Selfish element maintains sex in natural populations of a parasitoid wasp. Proc Biol Sci 268: 617-622.

Weeks AR, Breeuwer JA (2001). Wolbachia-induced parthenogenesis in a genus of phytophagous mites. Proc Biol Sci 268: 2245-2251.

Werren JH (1997). Biology of Wolbachia. Annu Rev Entomol 42: 587-609.

Zchori-Fein E, Faktor O, Zeidan M, Gottlieb Y, Czosnek H, Rosen D (1995). Parthenogenesis-inducing microorganisms in Aphytis (Hymenoptera: Aphelinidae). Insect Mol Biol 4: 173-178.

Zchori-Fein E, Roush RT, Hunter MS (1992). Male production induced by antibiotic treatment in Encarsia formosa (Hymenoptera, Aphelinidae), an asexual species. Experientia 48: 102-105.

Supplementary Information accompanies the paper on Heredity website (http://www.nature.com/hdy) 